Published in final edited form as:

J Neurooncol. 2014 May ; 118(1): 61-72. doi:10.1007/s11060-014-1419-0.

\title{
Expression of PRMT5 correlates with malignant grade in gliomas and plays a pivotal role in tumor growth in vitro
}

\author{
Xiaosi Han, \\ Department of Neurology, The University of Alabama at Birmingham, FOT 1020, 1530 3rd Ave S, \\ Birmingham, AL 35294-3410, USA xhan@uab.edu \\ Birmingham Veterans Affairs Medical Center, Birmingham, AL 35295, USA

\section{Rong Li,} \\ Department of Pathology, The University of Alabama at Birmingham, Birmingham, AL \\ 35294-3410, USA

\section{Wenbin Zhang,} \\ Department of Neurology, The University of Alabama at Birmingham, FOT 1020, 1530 3rd Ave S, \\ Birmingham, AL 35294-3410, USA

\section{Xiuhua Yang,} \\ Department of Neurology, The University of Alabama at Birmingham, FOT 1020, 1530 3rd Ave S, \\ Birmingham, AL 35294-3410, USA
}

\section{Crystal G. Wheeler,}

Department of Neurology, The University of Alabama at Birmingham, FOT 1020, 1530 3rd Ave S, Birmingham, AL 35294-3410, USA

\section{Gregory K. Friedman,}

Division of Hematology/Oncology, Department of Pediatrics, The University of Alabama at

Birmingham, Birmingham, AL 35294-3410, USA

\section{Paula Province,}

Department of Neurology, The University of Alabama at Birmingham, FOT 1020, 1530 3rd Ave S, Birmingham, AL 35294-3410, USA

\section{Qiang Ding,}

Division of Pulmonary Medicine, Department of Medicine, The University of Alabama at Birmingham, Birmingham, AL 35294-3410, USA

\section{Zhiying You,}

Department of Preventive Medicine, The University of Alabama at Birmingham, Birmingham, AL 35294-3410, USA

Hassan M. Fathallah-Shaykh,

\footnotetext{
(C) Springer Science+Business Media New York 2014

Correspondence to: Xiaosi Han.

Conflict of interest None of the authors has a conflict of interest to declare.
} 
Department of Neurology, The University of Alabama at Birmingham, FOT 1020, 1530 3rd Ave S, Birmingham, AL 35294-3410, USA

\author{
G. Yancey Gillespie, \\ Department of Neurosurgery, The University of Alabama at Birmingham, Birmingham, AL \\ 35294-3410, USA
}

\title{
Xinyang Zhao,
}

Department of Biochemistry and Molecular Genetics, The University of Alabama at Birmingham, Birmingham, AL 35294-3410, USA

Peter H. King, and

Birmingham Veterans Affairs Medical Center, Birmingham, AL 35295, USA

\section{Burt Nabors}

Department of Neurology, The University of Alabama at Birmingham, FOT 1020, 1530 3rd Ave S, Birmingham, AL 35294-3410, USA

\section{Abstract \\ Protein arginine methyltransferase 5 (PRMT5) catalyzes the formation of $\omega$-NG,N'G-symmetric dimethylarginine residues on histones as well as other proteins. These modifications play an important role in cell differentiation and tumor cell growth. However, the role of PRMT5 in human glioma cells has not been characterized. In this study, we assessed protein expression profiles of PRMT5 in control brain, WHO grade II astrocytomas, anaplastic astrocytomas, and glioblastoma multiforme (GBM) by immunohistochemistry. PRMT5 was low in glial cells in control brain tissues and low grade astrocytomas. Its expression increased in parallel with malignant progression, and was highly expressed in GBM. Knockdown of PRMT5 by small hairpin RNA caused alterations of p-ERK1/2 and significantly repressed the clonogenic potential and viability of glioma cells. These findings indicate that PRMT5 is a marker of malignant progression in glioma tumors and plays a pivotal role in tumor growth.}

\section{Keywords}

Diffuse astrocytoma; Anaplastic astrocytoma; Glioblastoma; PRMT5; ERK1/2

\section{Introduction}

Protein arginine methyltransferase (PRMT)-5 belongs to a family of enzymes that transfer a methyl group from $\mathrm{S}$-adenosylmethionine to the arginine side-chains of histones and other proteins [1]. PRMT members are divided into four types (I-IV) based on patterns of arginine methylation. PRMT5 is a type II enzyme and generates $\omega$-NG-monomethyl and $\omega-\mathrm{NG}, \mathrm{N}^{\prime}$ Gsymmetric di-methylarginine residues. PRMT5 plays a role in cell signaling, chromatin remodeling, and control of gene expression through its association with MEP50 and the subsequent methylation of many cytoplasmic and nuclear substrates, including histone, nucleolin, p53, eIF4E, EGFR, and C-RAF [2-7]. PRMT5 is emerging as an important enzyme involved in tumorigenesis and stem cell maintenance. Upregulation of PRMT5 is found in a number of cancers including lymphoma, gastric cancer, and germ cell tumors and 
has been linked to tumor cell growth and poor prognosis in breast and ovarian cancers [813]. PRMT5 promotes tumor progression by stimulating cell cycle progression, proliferation, and inhibition of apoptosis [6, 8, 14-17]. PRMT5 also plays an important role in stem cell maintenance through its suppression of differentiation genes [18]. During mouse embryonic development, the expression and subcellular localization of PRMT5 in the brain are linked specifically to neural cell fate determination [19]. Suppression of PRMT5 is required for neural stem cells to differentiate into neurons, which subsequently migrate from the subventricular zone to the cerebral cortex $[19,20]$.

Based on this background, we hypothesized that PRMT5 would be linked to the malignant phenotype in glioma tumors. Glioblastoma multiforme (GBM) is the most common and most aggressive primary brain tumor in adults with a median survival of 15-17 months [2123]. Identification of novel molecular targets is needed for development of effective therapy. Here, we analyzed PRMT5 expression in primary gliomas and found a strong correlation with tumor grade. Knockdown of PRMT5 in glioma cells caused a significant anti-growth effect associated with changes in activation dynamics of Erk1/2, indicating that PRMT5 plays an important role in the malignant phenotype of this tumor.

\section{Materials and methods}

\section{Tumor samples}

A total of 60 glioma specimens (20 specimens each of WHO grade II diffuse astrocytoma, WHO grade III anaplastic astrocytoma, and WHO grade IV GBM), and 10 control brains (resected from patients with temporal lobe epilepsy) were obtained from the Division of Neuropathology. The study was approved by the Institutional Review Board at the University of Alabama at Birmingham. All specimens were obtained at the time of first surgical resection.

\section{Immunohistochemistry}

Sections were deparaffinized by sequential treatment with citrisolv and isopropanol and rehydrated with water. Antigens were retrieved in citrate buffer in a rice steamer for $20 \mathrm{~min}$. Sections were treated in $3 \% \mathrm{H}_{2} \mathrm{O}_{2}$ for 30 min to quench superoxidase, and were then blocked (5\% goat serum, $0.3 \%$ Triton X-100 in PBS) and reacted with anti-PRMT5 (1:100, Santa Cruz Biotechnology, USA) overnight at $4{ }^{\circ} \mathrm{C}$. Tissues were incubated with peroxidase micropolymers conjugated secondary antibody (Impress antigoat 1:10, Vector Labs, USA) at room temperature for $1 \mathrm{~h}$ and were then washed. Biotin tyramide (1:400, Perk-inElmer, USA) was added to the section and was incubated for 10 min to amplify the signals. Slides were washed before incubation with $\mathrm{ABC}$ mix (prepared according to manufacturer's instruction, Vector Labs) for $30 \mathrm{~min}$. Sections were washed and reacted with 3,3'diaminobenzidine (DAB) substrate until the desired signal intensity developed. Slides were counter stained with hematoxylin and sealed with CytoSeal 60 (Thermo Scientific, USA). An H-Score was calculated based on scoring of stained cells according to published methods [24]. A semi-quantitative intensity scale ranging from 0 for no staining, 1 for weak staining, 2 for intermediate staining, and 3 for intense staining was used. The following formula: $\mathrm{H}-$ 
Score $=(\%$ at 0$) * 0+(\%$ at $1+) * 1+(\%$ at $2+) * 2+(\%$ at $3+) * 3$ was used to generate the H-Score.

\section{Immunofluorescent labeling of paraffin-embedded tissue section and fluorescence intensity measurements}

The method for immunofluorescent labeling is based on the protocol described in detail by Robertson et al. [25] with minor modifications. Briefly, slides were dewaxed by sequential treatment with xylene and ethanol and were then rehydrated. Antigens were retrieved in target retrieval solution (Dako) by incubating the slides at $94-96{ }^{\circ} \mathrm{C}$ for $20 \mathrm{~min}$. Tissue sections were blocked in PBS containing $2 \%$ each of BSA and horse serum $\left(25^{\circ} \mathrm{C}, 60 \mathrm{~min}\right)$ and reacted with goat anti-PRMT5 $(1: 100)$ at $4{ }^{\circ} \mathrm{C}$ overnight. Sections were washed, and incubated with Alexfluor-488 or Alexfluor-594 conjugated donkey anti-goat IgG (1:1000, Invitrogen $)\left(25^{\circ} \mathrm{C}, 60 \mathrm{~min}\right)$. For double labeling, slides were washed, and reacted with a second primary antibody (rabbit anti-GFAP, 1:200, Dako; rabbit anti-symmetric dimethylArginine (SYM10), 1:100; mouse anti-NeuN (Mab377), 1:100, Millipore) at $4{ }^{\circ} \mathrm{C}$ overnight, followed by incubation with Alexfluor-488 conjugated goat anti-rabbit $\operatorname{IgG}(1: 1,000)$ or Alexfluor-594 conjugated goat anti-mouse $\operatorname{IgG}\left(22^{\circ} \mathrm{C}, 60 \mathrm{~min}\right)$. After rinsing in PBS, sections were then stained with DAPI (50 $\mu \mathrm{g} / \mathrm{ml}$ in PBS) for $2 \mathrm{~min}$, and cover slips were mounted in Vectashield $\mathrm{H}-1000$ (Vector Laboratories) and sealed with nail varnish. Sections were visualized under an Olympus fluorescence microscope equipped with a digital camera. Monochrome images from each color channel were acquired separately and then merged. Fluorescence images were processed using Adobe Photoshop (Adobe Systems, USA).

Fluorescence intensity was measured on digital images of specimens simultaneously stained for SDMA and PRMT5 immunoreactivity. Measurement of mean cell brightness is based on methods previously described [26]. On the fluorescence microscope, the background fluorescence of the tissue of each image was set to a barely detectable level by adjusting the gain of the charge-coupled device camera, and then the image was captured. RGB images were converted to 8-bit gray scale images (fluorescence intensity range: 0 to $<255$ ) using ImageJ software (http://rsb.info.nih.gov/ij). The fluorescence intensity was measured and the average brightness of all cell-related pixels was calculated. This value was obtained as follows: (a) a cell of interest was delineated; (b) an adjacent same sized area was chosen as background; (c) the net cell-related fluorescent intensity was obtained by subtracting the background; (d) the average fluorescence intensity was calculated by dividing the net cellrelated intensity by the total number of pixels. The mean value was calculated for four images of tissue from each control brain $(n=10)$.

\section{Plasmids, shRNAs, lentiviruses and cell lines}

Lentiviral gene transfer vector pLKO-Tet-On, packaging (psPAX2) and envelope (pMD2.G) plasmids were purchased from Addgene (Cambridge, USA). One pair of control (scrambled) shRNA and two pairs of oligonucleotides targeting different regions of the PRMT5 gene (target sequence \#1: 5'-GGGACTGGAATACGCTAATTG-3', \#2: 5'-GGTC TTCCAGCTTTCCTGCTG-3') were synthesized, annealed, and inserted into pLKO-Tet-On at $A g e \mathrm{I}$ and $E c o$ RI sites. Viruses were generated according to methods described by Wiederschain et al. [27]. Glioma cell lines (U373MG and LN229) were plated in 6-well 
plates and grown to $60 \%$ confluency before lentiviruses were introduced at a multiplicity of infection (MOI) of 0.5. Cells were incubated with the viruses for $24 \mathrm{~h}$ before the medium was replaced with puromycin $(1 \mu \mathrm{g} / \mathrm{ml})$ containing medium. Puromycin selection was completed after 3 days of treatment and the resistant cells were used for further experiments. Doxycycline $(200 \mathrm{ng} / \mathrm{ml})$ inducible knockdown of PRMT5 was examined at different time points after exposure to doxycycline.

\section{Immunoblotting}

Cultured cells were lysed in M-PER mammalian protein extraction buffer in the presence of 19 Halt proteinase and phosphatase inhibitor cocktail $(100 \mu \mathrm{g} / \mathrm{mL}$ aprotinin, $10 \mu \mathrm{g} / \mathrm{mL}$ leupeptin, $2 \mathrm{mg} / \mathrm{ml}$ AEBSF hydrochloride, $50 \mu \mathrm{g} / \mathrm{ml}$ Bestatin, $200 \mu \mathrm{g} / \mathrm{ml} \mathrm{E}-64,100 \mathrm{mg} / \mathrm{ml}$ EDTA, $10 \mu \mathrm{g} / \mathrm{ml}$ Pepstatin A, Thermo Scientific). Protein concentrations were determined by BCA protein assay kit (Pierce). Equivalent amounts of protein $(30 \mu \mathrm{g})$ were resolved on an $8-16 \%$ gradient Precise Protein Gel (disulfide-reduced SDS-PAGE gel, Thermo Scientific), transferred to a nitrocellulose membrane (Millipore Corp), blocked with $5 \%$ nonfat milk in TBST $\left(1 \mathrm{~h}, 22{ }^{\circ} \mathrm{C}\right)$, and reacted with the primary antibody at $4{ }^{\circ} \mathrm{C}$ overnight followed by a secondary antibody conjugated to horseradish peroxidase (Sigma). Total Akt (Akt), phospho-Akt (p-Akt, Ser473) antibodies were purchased from Cell Signaling Technology (Beverley, MA). Mouse anti-ERK1/2, rabbit anti-p-ERK1/2, and mouse antiactin antibodies were purchased from Millipore, and used at $0.1 \mu \mathrm{g} / \mathrm{ml}$ (goat anti-PRMT5) or $0.05 \mu \mathrm{g} / \mathrm{ml}$ (mouse anti-ERK1/2, rabbit anti-p-ERK1/2, and mouse anti-actin). Membranebound antibodies were detected by enhanced chemiluminescence (Biosciences). For densitometric analysis of band intensity, a specific band on the ECL-developed film was subjected to densitometric analysis as described previously [28]. The densitometric readings were pooled and averaged from at least three independent experiments. The background of densitometric reading on the ECL-developed film was subtracted.

\section{Cell viability and colony formation assays}

The cell lines U373MG and LN229 were cultured in glioma medium (DMEM/F12 50:50, 9 $\%$ FBS, $1 \mathrm{mM}$ glutamine, 10/ml penicillin and $10 \mu \mathrm{g} / \mathrm{ml}$ streptomycin) and passaged twice per week. For Viability assay, 2,000 cells were plated in each well of a 96-well plate in growth medium containing $2 \%$ FBS with or without doxycycline. Viable cells were determined on day 4 by MTS colorimetric assay using the CellTiter $966^{\circledR}$ Aqueous One Solution kit (Promega) following the protocol recommended by the manufacturer. The MTS (3-(4,5-dimethylthiazol-2-yl)-5-(3-carboxymethoxyphenyl)-2-(4-sulfophenyl)-2Htetrazolium, inner salt) assay is based on the conversion of tetrazolium salt into a colored, aqueous soluble formazan product by mitochondrial activity of viable cells at $37^{\circ} \mathrm{C}$. The amount of formazan produced by dehydrogenase enzymes is directly proportional to the number of living cells in culture and was measured at $490 \mathrm{~nm}$ in a Tecan Infinite M200 microplate reader (Tecan, Germany). The amount of viable cells in control and doxycycline treated cultures was determined by taking the average of three experiments. For colony formation assays, cells were seeded at 200 cells per well in 6-well plates and incubated in the absence or presence of tetracycline for 14 days. Cells were fixed with $5 \%$ paraformaldehyde for $10 \mathrm{~min}$ and stained with crystal violet $(0.05 \%$ in $0.2 \mathrm{M}$ sodium 
borate, $\mathrm{pH}$ 9.0) for $20 \mathrm{~min}$. Colonies were visualized and those with 20 cells or more were counted.

\section{Statistical analyses}

Experimental data were analyzed using one-way analysis of variance (ANOVA) or twotailed Student's $t$ test to determine the statistical significance (Sigma Plot 2000, SPSS Inc). If a significant difference was found by ANOVA, the Tukey-Kramer multiple comparison procedure was then used to determine which means differ. The results were expressed as mean \pm standard errors (SEM). All experiments were repeated at least three times. A $p$ value $<0.05$ was considered to be statistically significant.

\section{Results}

\section{PRMT5 is expressed in the cortex of brain}

PRMT5 is dynamically regulated during mouse brain development, but its expression and function in human brain has never been reported. We examined PRMT5 expression in human brain tissues resected from patients with temporal lobe epilepsy. Ten patient samples were assessed, and representative results are shown in Fig. 1. We used paraffin-embedded colon cancer as a positive control for immunohistochemistry staining (Fig. 1a, b), and the staining pattern is consistent with previously published findings [29]. Non-specific staining was minimal in the cerebral cortex and brain deep white matter in the absence of primary antibody (Fig. 1c, g). PRMT5 was detected in a significant proportion of cortical cells, many with morphological features of neurons (Fig. 1d - f). In contrast, PRMT5 was very low in the vast majority of cells in the deep white matter (Fig. 1h, i).

\section{PRMT5 expression is high in neuronal cells}

Based on the morphological features of PRMT5-postive cells shown above, we hypothesized that PRMT5 is predominantly expressed in neuronal cells in human brain. Colocalization studies with NeuN and GFAP confirmed this hypothesis (Fig. 2). NeuN was found in the nucleus of neuronal cells, while absent in adjacent cells (Fig. 2e, f). PRMT5 was detected in the nuclei of NeuN-positive cells (Fig. $2 \mathrm{~g}$, h), but not in the majority of GFAP positive cells (Fig. $2 \mathrm{~m}-\mathrm{p}$ ).

\section{PRMT5 is an active enzyme in human brain neuroepithelial cells}

To demonstrate whether PRMT5 is active in neuroepithelial cells, we stained for symmetrical dimethylated arginine (SDMA), the enzymatic product of PRMT5, using antisym10 antibody (Fig. 3). SDMA was detected with variable intensity in the nuclei of many cells (Fig. 3d). PRMT5 expressing cells were strongly positive for SDMA, whereas cells negative for PRMT5 stained weakly for SDMA (Fig. 3d - f). The relative fluorescence intensity of SDMA was measured and found to be significantly higher in PRMT5 positive cells (G). These results demonstrate that the end product of PRMT5 enzymatic activity is present in cells expressing PRMT5 in human brains. The detection of SDMA in cells that are negative for PRMT5 could result from the expression of other PRMT family members, such as PRMT7, which have similar enzymatic properties [30]. Taken together, these results demonstrate that PRMT5 is present as an active enzyme in neurons of the cerebral cortex. 


\section{PRMT5 expression correlates with grade of malignancy in astrocytomas}

We next examined the expression of PRMT5 in different grades of gliomas, including WHO grade II, grade III, and grade IV astrocytomas. Twenty cases each were examined by IHC and the results are summarized in Table 1.

Representative images of the reactivity in each grade are shown in Fig. 4. In grade II astrocytomas, the cellularity was increased with occasional nuclear atypia without mitotic activity. PRMT5 expression was very low in the majority of tumor cells (Fig. 4c, d). However, very few isolated PRMT5 positive cells were present in each specimen and small pockets of PRMT5 positive cells consisting of 5-15 cells were identified in a few cases (not shown). In anaplastic astrocytomas, typical features of increased cellularity with distinct nuclear atypia and mitotic activity without multilayered microvessels or necrosis were seen. PRMT5 was detected in many cells of anaplastic astrocytomas throughout the specimen (Fig. 4e, f). In GBMs, poorly differentiated pleomorphic astrocytic tumor cells with marked nuclear atypia and brisk mitotic activity were observed along with prominent microvasculature proliferation and/or necrosis. Expression of PRMT5 was found in all of the GBM specimens with heterogeneous distribution among different tumor cells (Fig. 4g, h). Cells that were strongly positive for PRMT5 were adjacent to negative cells. PRMT5 was predominantly nuclear in GBM tumor cells with some cytoplasmic distribution as well. Semiquantitative analysis of immunostained sections by H-Scoring was performed [24]. The H-Score of PRMT5 correlated with tumor grade with GBMs showing the highest score, followed by anaplastic astrocytomas and then grade II astrocytomas (Fig. 4i). These results suggest that PRMT5 may serve as a marker for tumor aggressiveness and grade.

Expression of PRMT5 in different grades of astrocytomas was further examined by immunofluorescent studies (Fig. 5). Consistent with the immunohistochemical studies, PRMT5 was very low in diffuse astrocytomas (Fig. 5a), moderate in anaplastic astrocytomas (Fig. 5b) and high in GBM specimens (Fig. 5c). PRMT5 appeared predominantly located in the nuclei of GBM cells, in contrast to a more diffuse distribution in cells of anaplastic astrocytomas. PRMT5 expressing cells were positive for GFAP, indicating tumors of astrocytic origin.

\section{Knockdown of PRMT5 changes the dynamics of ERK1/2 activation and inhibits colony formation and growth of glioma cell lines}

To determine the functional role of PRMT5 in glioma cells, we first analyzed its expression in several commonly used glioma cell lines including U251MG, U373MG, LN229, and U87MG by Western blot. We detected PRMT5 expression in all the glioma cell lines (not shown). We next transduced U373 and LN229 cells with lentiviruses harboring tetinducible shRNAs (or scrambled control) targeting discrete regions of the PRMT5 mRNA. Stable clones were selected and induced with doxycycline (200 ng/ml). PRMT5 expression was assessed by Western blot at different time points after induction. The levels of PRMT5 were unaffected by the control shRNA and were decreased by the targeting shRNAs (Fig. 6a, b). By day 6 , the levels of PRMT5 diminished to less than 20 and $10 \%$ of control by shRNA\#1 and shRNA\#2, respectively (Fig. 6c, d). We next examined whether PRMT5 regulates Akt or ERK1/2, two of the most frequently altered signaling pathways in GBM. U373MG clones 
containing shRNAs were grown in the presence or absence of doxycycline and serumstarved for $24 \mathrm{~h}$. The culture medium was then replaced with serum containing medium ( 9 $\%$ FBS) and lysates were prepared at different time points for western blot analysis (Fig. 6e, f). P-Akt was not affected by PRMT5 knockdown. In contrast, the p-ERK1/2 peaked at 30 min and gradually declined to baseline over $48 \mathrm{~h}$ in the controls. With PRMT5 knockdown in shRNA\#1 or shRNA\#2, prolonged activation of ERK1/2 was observed (Fig. $6 \mathrm{~g}, \mathrm{~h}$ ). Prolonged activation of ERK1/2 by PRMT5 knockdown was also observed in LN229 (data not shown). These results demonstrated that PRMT5 regulates the activation dynamics of ERK1/2 in serum containing medium.

The role of PRMT5 in tumorigenesis was assessed with a colony-formation assay. Glioma cells harboring shRNAs were plated in medium with or without doxycycline. After 14 days, the colonies were fixed and quantified (Fig. 7a). The number of colonies (defined as $\geq 20$ cells) was expressed as a percent of the vehicle control. Knockdown of PRMT5 significantly reduced the numbers of colonies formed in both glioma cell lines compared to uninduced cells or the shRNA control, indicating that PRMT5 plays an important role in colony formation (Fig. 7b). To determine the role of PRMT5 in cell growth and survival, we performed a viability assay following PRMT5 knockdown. Cells were induced with doxycycline or vehicle for 6 days and then assessed for viability by the MTS assay (Fig. 7c). The percentage of viable cells was significantly reduced in both PRMT5 shRNA clones after doxycycline induction, but not in control shRNA cells. These results demonstrate that PRMT5 plays a significant role in promoting malignant properties of gliomas.

\section{Discussion}

PRMT5 plays a critical role in normal embryonic development, cellular differentiation, and pathological processes such as neoplasms [29]. To address the role of PRMT5 in gliomas, we assessed expression of PRMT5 in low-grade diffuse astrocytoma, anaplastic astrocytoma, glioblastoma, and control brain. PRMT5 was undetectable or low in astrocytic cells, while it was expressed in neuronal cells of control brains. PRMT5 expression correlated with the degree of malignancy, ranging from very low expression in diffuse astrocytomas to high expression in GBM tumors, suggesting that it may be a marker of tumor aggressiveness and grade in astrocytomas. Finally, we showed that knockdown of PRMT5 altered the activation profile of ERK1/2 and inhibited glioma cell growth, suggesting a functional role in gliomagenesis.

PRMT5 is emerging as a critical regulator of cellular stemness and differentiation. Its role in preserving the less-differentiated cellular state has been demonstrated in primordial germ cells, erythrocyte progenitors, embryonic stem cells, and neural stem cells [18, 20, 31, 32]. At the molecular level, symmetric dimethylation of arginine R3 on histones by PRMT5 is associated with transcriptional repression of genes involved in neural stem cell differentiation and neuronal lineage determination [33]. For example, PRMT5 is recruited to histones through interaction with Schwann Cell Factor 1 (SC1/PRDM4) and serves as an epigenetic enzyme essential for maintaining neural stem cells [20]. During mouse embryonic development, the expression and subcellular localization of PRMT5 in the brain are linked specifically to neural epithelial cell fate determination [19]. Suppression of 
PRMT5 is required for neural stem cells to differentiate into neurons which subsequently migrate from the subventricular zone to the cerebral cortex $[19,20]$. On the other hand, PRMT5 expression gradually increases during postnatal brain development in mice and is required for oligodendrocyte differentiation [34]. PRMT5 is dynamically regulated during brain development and neuroepithelial differentiation. The differential expression of PRMT5 in different grades of astrocytomas may reflect the degree of de-differentiation of these tumors. In highly differentiated diffuse astrocytomas, PRMT5 is either undetectable or very low. Glioma cells in anaplastic astrocytomas are less differentiated and showed some expression of PRMT5, whereas GBM cells had the highest levels of expression. These results raise the possibility that PRMT5 may be important in maintaining the dedifferentiation state of glioma cells. Given the importance of glioma stem cells in cancer initiation, propagation, and treatment resistance and recurrence, further investigation into the role of PRMT5 in glioma stem cells is warranted. One caveat is that we cannot rule out the possibility that some of the PRMT5 expressing cells include reactive astrocytes in glioma specimens. Since GBMs consist of cells at various stages of differentiation [35, 36], variations in PRMT5 expression in these cells is not surprising.

EGFR amplification and overexpression are well documented genomic changes in up to 60 $\%$ of GBM tumors [37]. The aberrant activation of EGFR/ERK1/2 has been well characterized as a major signal for gliomagenesis [38]. We examined whether PRMT5 regulates this important pathway and found that knockdown of PRMT5 altered the activation dynamics of ERK1/2. The observed modulation of p-ERK1/2 was associated with inhibition of colony formation and tumor cell viability. Prior studies have shown that PRMT5 methylates EGFR and RAF resulting in their inactivation or degradation [3, 7]. The methylation events are important in restricting growth factor initiated signaling and allow downstream signal p-ERK1/2 to terminate in a timely manner after growth factor exposure $[3,7]$. Available evidence suggests that the duration of ERK activation is critical for cell signaling decisions [39]. Sustained overactivation of ERK1/2 induces autophagy and cell death in GBM cells [40], inhibits tumor cell growth and promotes differentiation in PC12 cells [7, 41], and causes growth arrest of small cell lung cancer cells [42]. It is possible that PRMT5-mediated regulation of ERK1/2 signaling contributes to glioma cell growth. Alternatively, PRMT5 knockdown could affect methylation status of other substrates including histone $\mathrm{H} 3$ and transcription factor E2F which can affect tumor cell growth [17, 43].

In conclusion, we find that PRMT5 is expressed in astrocytomas and correlates with tumor grade. PRMT5 promotes the malignant phenotype of glioma cells, possibly through modulation of the ERK1/2 pathway. The results suggest that PRMT5 may be a target for glioma therapy.

\section{Acknowledgments}

NIH NCI Grants P30CA013148 (UAB Comprehensive Cancer Center Core Support Grant); P50CA097247, P20CA151129 (G. Y. Gillespie); St. Baldrick's Foundation (G. K. Friedman); VA Merit Review (P. H. King). 


\section{References}

1. Yang Y, Bedford MT. Protein arginine methyltransferases and cancer. Nat Rev. 2013; 13(1):37-50.

2. Friesen WJ, Paushkin S, Wyce A, et al. The methylosome, a 20S complex containing JBP1 and pICln, produces dimethylarginine-modified Sm proteins. Mol Cell Biol. 2001; 21(24):8289-8300. [PubMed: 11713266]

3. Hsu JM, Chen CT, Chou CK, et al. Crosstalk between Arg 1175 methylation and Tyr 1173 phosphorylation negatively modulates EGFR-mediated ERK activation. Nat Cell Biol. 2011; 13(2): 174-181. [PubMed: 21258366]

4. Jansson M, Durant ST, Cho EC, et al. Arginine methylation regulates the p53 response. Nat Cell Biol. 2008; 10(12):1431-1439. [PubMed: 19011621]

5. Teng Y, Girvan AC, Casson LK, et al. AS1411 alters the localization of a complex containing protein arginine methyltransferase 5 and nucleolin. Cancer Res. 2007; 67(21):10491-10500. [PubMed: 17974993]

6. Scoumanne A, Zhang J, Chen X. PRMT5 is required for cell-cycle progression and p53 tumor suppressor function. Nucleic Acids Res. 2009; 37(15):4965-4976. [PubMed: 19528079]

7. Andreu-Perez P, Esteve-Puig R, Esteve-Puig R, de Torre-Minguela C, et al. Protein arginine methyltransferase 5 regulates ERK1/2 signal transduction amplitude and cell fate through CRAF. Sci Signal. 2011; 4(190):ra58. [PubMed: 21917714]

8. Pal S, Baiocchi RA, Byrd JC, et al. Low levels of miR-92b/ 96 induce PRMT5 translation and H3R8/H4R3 methylation in mantle cell lymphoma. EMBO J. 2007; 26(15):3558-3569. [PubMed: 17627275]

9. Wang L, Pal S, Sif S. Protein arginine methyltransferase 5 suppresses the transcription of the RB family of tumor suppressors in leukemia and lymphoma cells. Mol Cell Biol. 2008; 28(20):62626277. [PubMed: 18694959]

10. Kim JM, Sohn HY, Yoon SY, et al. Identification of gastric cancer-related genes using a cDNA microarray containing novel expressed sequence tags expressed in gastric cancer cells. Clin Cancer Res. 2005; 11(2 Pt 1):473-482. [PubMed: 15701830]

11. Eckert D, Biermann K, Nettersheim D, et al. Expression of BLIMP1/PRMT5 and concurrent histone $\mathrm{H} 2 \mathrm{~A} / \mathrm{H} 4$ arginine 3 dimethylation in fetal germ cells, CIS/IGCNU and germ cell tumors. BMC Dev Biol. 2008; 8:106. [PubMed: 18992153]

12. Powers MA, Fay MM, Factor RE, et al. Protein arginine methyltransferase 5 accelerates tumor growth by arginine methylation of the tumor suppressor programmed cell death 4 . Cancer Res. 2011; 71(16):5579-5587. [PubMed: 21700716]

13. Bao X, Zhao S, Liu T, et al. Overexpression of PRMT5 promotes tumor cell growth and is associated with poor disease prognosis in epithelial ovarian cancer. J Histochem Cytochem. 2013; 61(3):206-217. [PubMed: 23292799]

14. Tanaka H, Hoshikawa Y, Oh-hara T, et al. PRMT5, a novel TRAIL receptor-binding protein, inhibits TRAIL-induced apoptosis via nuclear factor-kappaB activation. Mol Cancer Res. 2009; 7(4):557-569. [PubMed: 19372584]

15. Yang M, Sun J, Sun X, et al. Caenorhabditis elegans protein arginine methyltransferase PRMT-5 negatively regulates DNA damage-induced apoptosis. PLoS Genet. 2009; 5(6):e1000514. [PubMed: 19521535]

16. Aggarwal P, Vaites LP, Kim JK, et al. Nuclear cyclin D1/ CDK4 kinase regulates CUL4 expression and triggers neoplastic growth via activation of the PRMT5 methyltransferase. Cancer Cell. 2010; 18(4):329-340. [PubMed: 20951943]

17. Pal S, Vishwanath SN, Erdjument-Bromage H, Tempst P, Sif S. Human SWI/SNF-associated PRMT5 methylates histone H3 arginine 8 and negatively regulates expression of ST7 and NM23 tumor suppressor genes. Mol Cell Biol. 2004; 24(21):9630-9645. [PubMed: 15485929]

18. Tee WW, Pardo M, Theunissen TW, et al. PRMT5 is essential for early mouse development and acts in the cytoplasm to maintain ES cell pluripotency. Genes Dev. 2010; 24(24):2772-2777. [PubMed: 21159818]

19. Chittka A. Dynamic distribution of histone $\mathrm{H} 4$ arginine 3 methylation marks in the developing murine cortex. PLoS One. 2010; 5(11):e13807. [PubMed: 21072194] 
20. Chittka A, Nitarska J, Grazini U, Richardson WD. Transcription factor positive regulatory domain 4 (PRDM4) recruits protein arginine methyltransferase 5 (PRMT5) to mediate histone arginine methylation and control neural stem cell proliferation and differentiation. J Biol Chem. 2012; 287(51):42995-43006. [PubMed: 23048031]

21. Jemal A, Murray T, Samuels A, et al. Cancer statistics, 2003. CA Cancer J Clin. 2003; 53(1):5-26. [PubMed: 12568441]

22. Ohgaki H, Kleihues P. Population-based studies on incidence, survival rates, and genetic alterations in astrocytic and oli-godendroglial gliomas. J Neuropathol Exp Neurol. 2005; 64(6): 479-489. [PubMed: 15977639]

23. Tran B, Rosenthal MA. Survival comparison between glioblastoma multiforme and other incurable cancers. J Clin Neurosci. 2010; 17(4):417-421. [PubMed: 20167494]

24. Ishibashi H, Suzuki T, Suzuki S, et al. Sex steroid hormone receptors in human thymoma. J Clin Endocrinol Metab. 2003; 88(5):2309-2317. [PubMed: 12727990]

25. Robertson D, Savage K, Reis-Filho JS, Isacke CM. Multiple immunofluorescence labelling of formalin-fixed paraffin-embedded (FFPE) tissue. BMC Cell Biol. 2008; 9:13. [PubMed: 18366689]

26. Inai T, Mancuso M, Hashizume $\mathrm{H}$, et al. Inhibition of vascular endothelial growth factor (VEGF) signaling in cancer causes loss of endothelial fenestrations, regression of tumor vessels, and appearance of basement membrane ghosts. Am J Pathol. 2004; 165(1):35-52. [PubMed: 15215160]

27. Wiederschain D, Wee S, Chen L, et al. Single-vector inducible lentiviral RNAi system for oncology target validation. Cell Cycle. 2009; 8(3):498-504. [PubMed: 19177017]

28. Han X, Stewart JE Jr. Bellis SL, et al. TGF-beta1 upregulates paxillin protein expression in malignant astrocytoma cells: requirement for a fibronectin substrate. Oncogene. 2001; 20(55): 7976-7986. [PubMed: 11753680]

29. Karkhanis V, Hu YJ, Baiocchi RA, et al. Versatility of PRMT5-induced methylation in growth control and development. Trends Biochem Sci. 2011; 36(12):633-641. [PubMed: 21975038]

30. Lee JH, Cook JR, Yang ZH, et al. PRMT7, a new protein arginine methyltransferase that synthesizes symmetric dimethylarginine. J Biol Chem. 2005; 280(5):3656-3664. [PubMed: 15494416]

31. Zhao Q, Rank G, Tan YT, et al. PRMT5-mediated methylation of histone H4R3 recruits DNMT3A, coupling histone and DNA methylation in gene silencing. Nat Struct Mol Biol. 2009; 16(3):304-311. [PubMed: 19234465]

32. Ancelin K, Lange UC, Hajkova P, et al. Blimp1 associates with Prmt5 and directs histone arginine methylation in mouse germ cells. Nat Cell Biol. 2006; 8(6):623-630. [PubMed: 16699504]

33. Bedford MT, Clarke SG. Protein arginine methylation in mammals: who, what, and why. Mol Cell. 2009; 33(1):1-13. [PubMed: 19150423]

34. Huang J, Vogel G, Yu Z, Almazan G, Richard S. Type II arginine methyltransferase PRMT5 regulates gene expression of inhibitors of differentiation/DNA binding Id 2 and Id 4 during glial cell differentiation. J Biol Chem. 2011; 286(52):44424-44432. [PubMed: 22041901]

35. Friedmann-Morvinski D, Bushong EA, Ke E, et al. Dedifferentiation of neurons and astrocytes by oncogenes can induce gliomas in mice. Science. 2012; 338(6110):1080-1084. [PubMed: 23087000]

36. Dunn GP, Rinne ML, Wykosky J, et al. Emerging insights into the molecular and cellular basis of glioblastoma. Genes Dev. 2012; 26(8):756-784. [PubMed: 22508724]

37. Verhaak RG, Hoadley KA, Purdom E, et al. Integrated genomic analysis identifies clinically relevant subtypes of glioblastoma characterized by abnormalities in PDGFRA, IDH1, EGFR, and NF1. Cancer Cell. 2010; 17(1):98-110. [PubMed: 20129251]

38. Krakstad C, Chekenya M. Survival signalling and apoptosis resistance in glioblastomas: opportunities for targeted therapeutics. Mol Cancer. 2010; 9:135. [PubMed: 20515495]

39. Marshall CJ. Specificity of receptor tyrosine kinase signaling: transient versus sustained extracellular signal-regulated kinase activation. Cell. 1995; 80(2):179-185. [PubMed: 7834738] 
40. Olsen BB, Svenstrup TH, Guerra B. Downregulation of protein kinase CK2 induces autophagic cell death through modulation of the mTOR and MAPK signaling pathways in human glioblastoma cells. Int J Oncol. 2012; 41(6):1967-1976. [PubMed: 23007634]

41. Pang L, Sawada T, Decker SJ, Saltiel AR. Inhibition of MAP kinase kinase blocks the differentiation of PC-12 cells induced by nerve growth factor. J Biol Chem. 1995; 270(23):1358513588. [PubMed: 7775407]

42. Ravi RK, Weber E, McMahon M, et al. Activated Raf-1 causes growth arrest in human small cell lung cancer cells. J Clin Investig. 1998; 101(1):153-159. [PubMed: 9421477]

43. Cho EC, Zheng S, Munro S, et al. Arginine methylation controls growth regulation by E2F-1. EMBO J. 2012; 31(7):1785-1797. [PubMed: 22327218] 

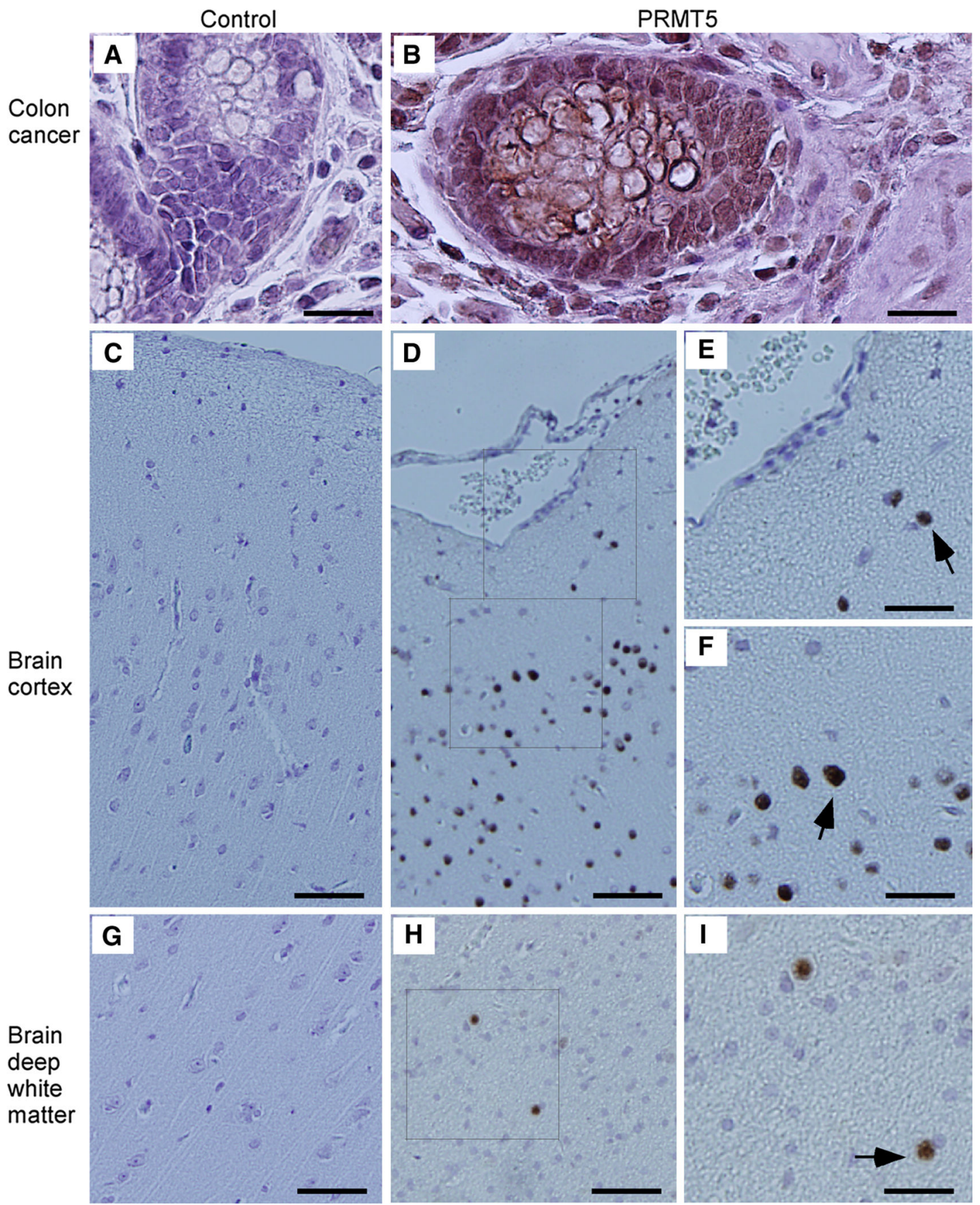

Fig. 1.

PRMT5 is expressed in the cortex of brain tissues. Tissue sections were immunostained with an anti-PRMT5 antibody in comparison to a negative control (no primary antibody). a, b PRMT5 was present in colon cancer which served as a positive control for the antibody. Scale bar 5 m. c, g Controls using secondary antibody alone showed only minimal background signal. Scale bar $20 \mu \mathrm{m}$. d-f Intense immunoreactivity of PRMT5 was seen in a select subset of cortical cells (arrows). Scale bar $20 \mu \mathrm{m}$ for $\mathbf{d}, 10 \mu \mathrm{m}$ for $\mathbf{e}$ and $\mathbf{f} . \mathbf{h}, \mathbf{i}$ PRMT5 was found in only a few cells in the deep white matter (arrow), but was 
undetectable in the majority of cells or the extracellular matrix. Scale bar $20 \mu \mathrm{m}$ for $\mathbf{h}$ and $10 \mu \mathrm{m}$ for $\mathbf{i}$ 

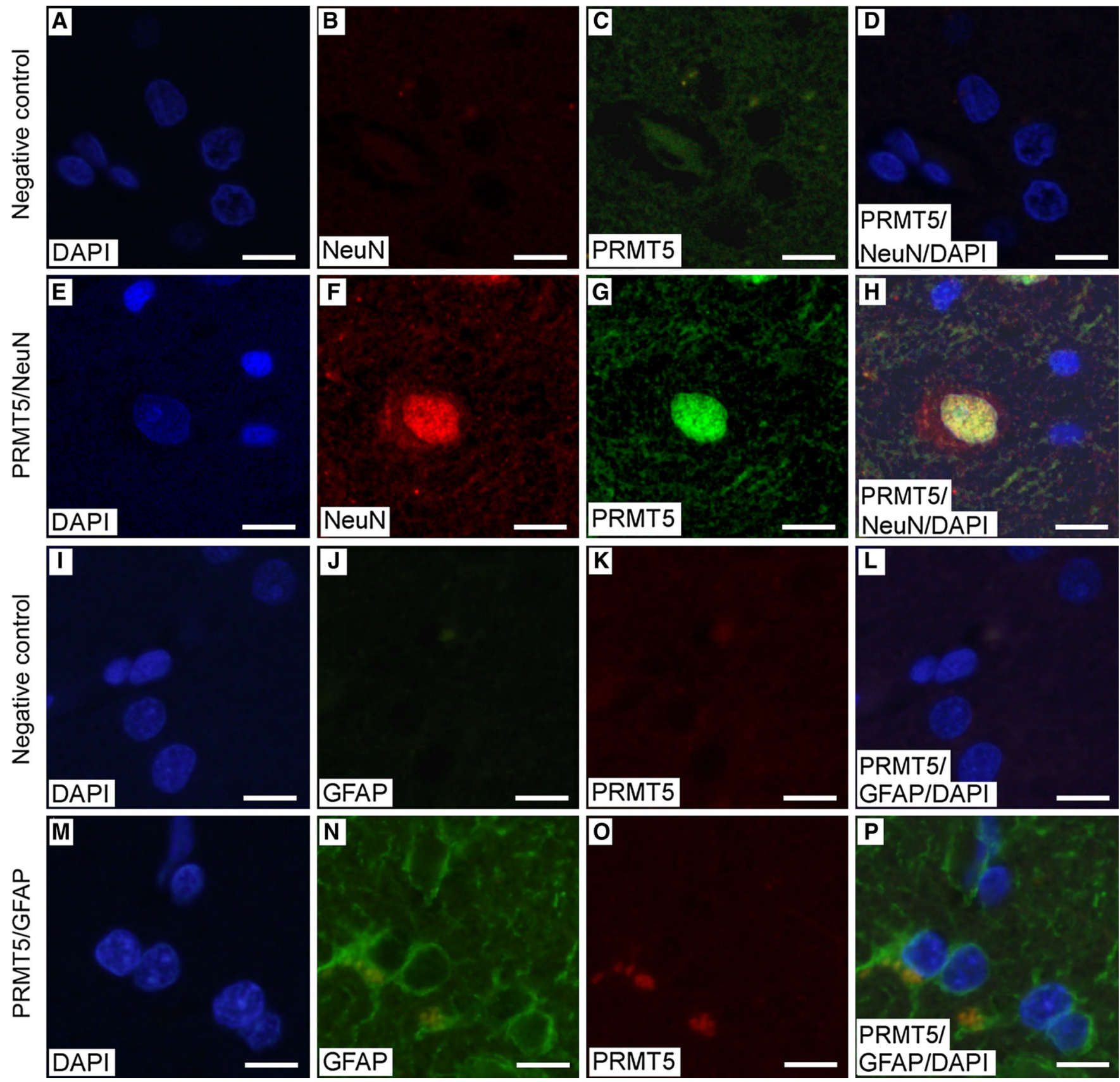

Fig. 2.

PRMT5 co-localizes with NeuN in human brain tissues. Tissue sections were immunostained with anti-PRMT5, anti-NeuN or anti-GFAP antibodies and were assessed by immunofluorescence. Scale bar $2 \mu \mathrm{m}$. a-d Immunofluorescence showed minimal background autofluorescence in negative controls where primary antibodies for PRMT5 and NeuN were absent. $\mathbf{e}-\mathbf{h}$ PRMT5 co-localized to the nuclei of cells positive for NeuN. $\mathbf{i}-\mathbf{l}$ Negative controls for PRMT5 and GFAP were shown. m-p PRMT5 was either undetectable or low in cells expressing GFAP 


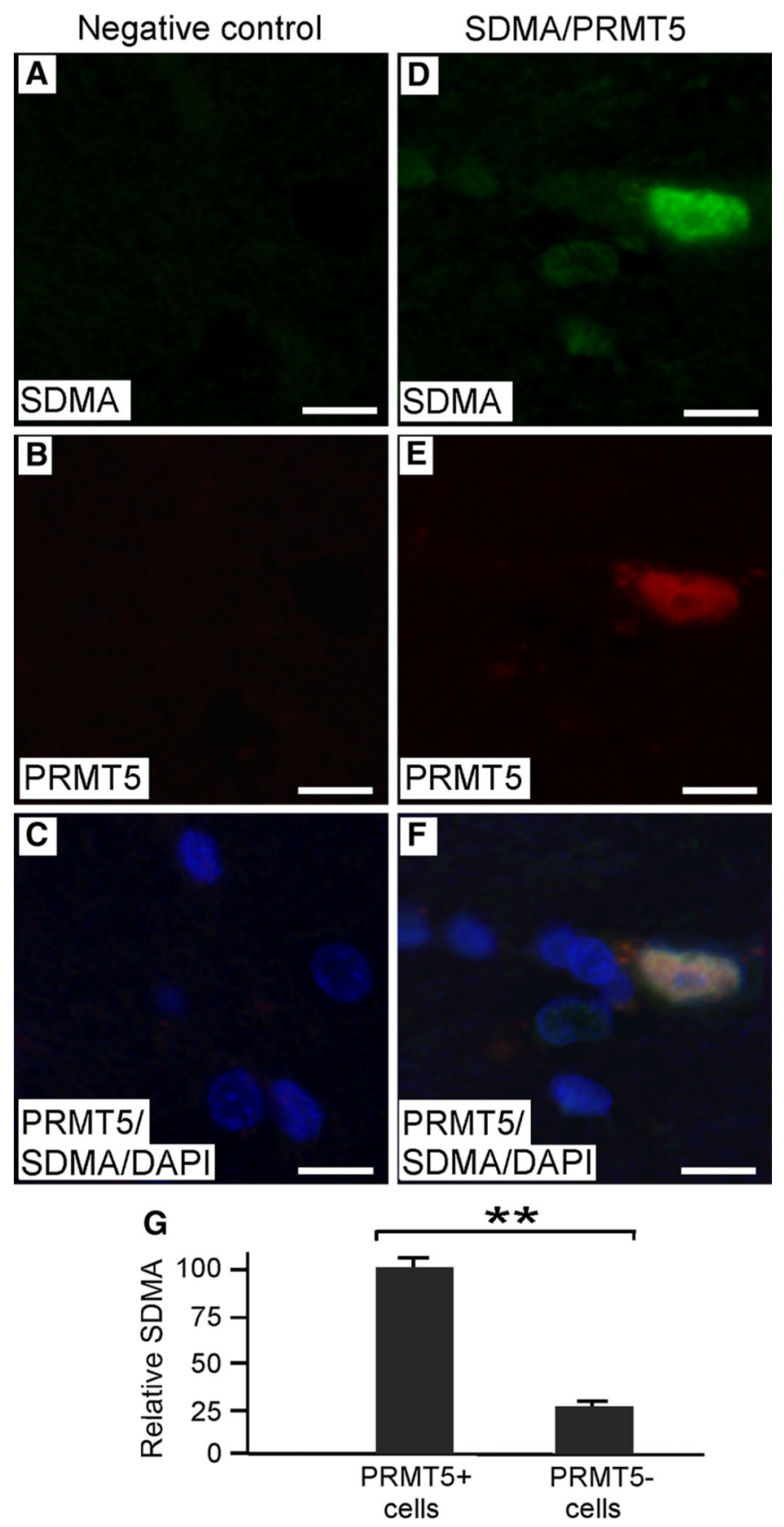

Fig. 3.

PRMT5 is an active enzyme in neuroepithelial cells of human brain tissues. Tissue sections were immunostained with anti-PRMT5 and anti-Sym10, which specifically recognizes symmetrical dimethylated arginine residue (SDMA), the end product of PRMT5. Scale bar $2 \mu \mathrm{m}$. a-c Background staining was minimal in the absence of primary antibody. $\mathbf{d}-\mathbf{f}$ Cells positive for PRMT5 were strongly positive for SDMA, whereas cells negative for PRMT5 were weakly positive for SDMA. g Relative fluorescence intensity of SDMA in cells 
positive for PRMT5 was significantly higher (3.6-fold) than that in PRMT5 negative cells. $* * p<0.01$ 


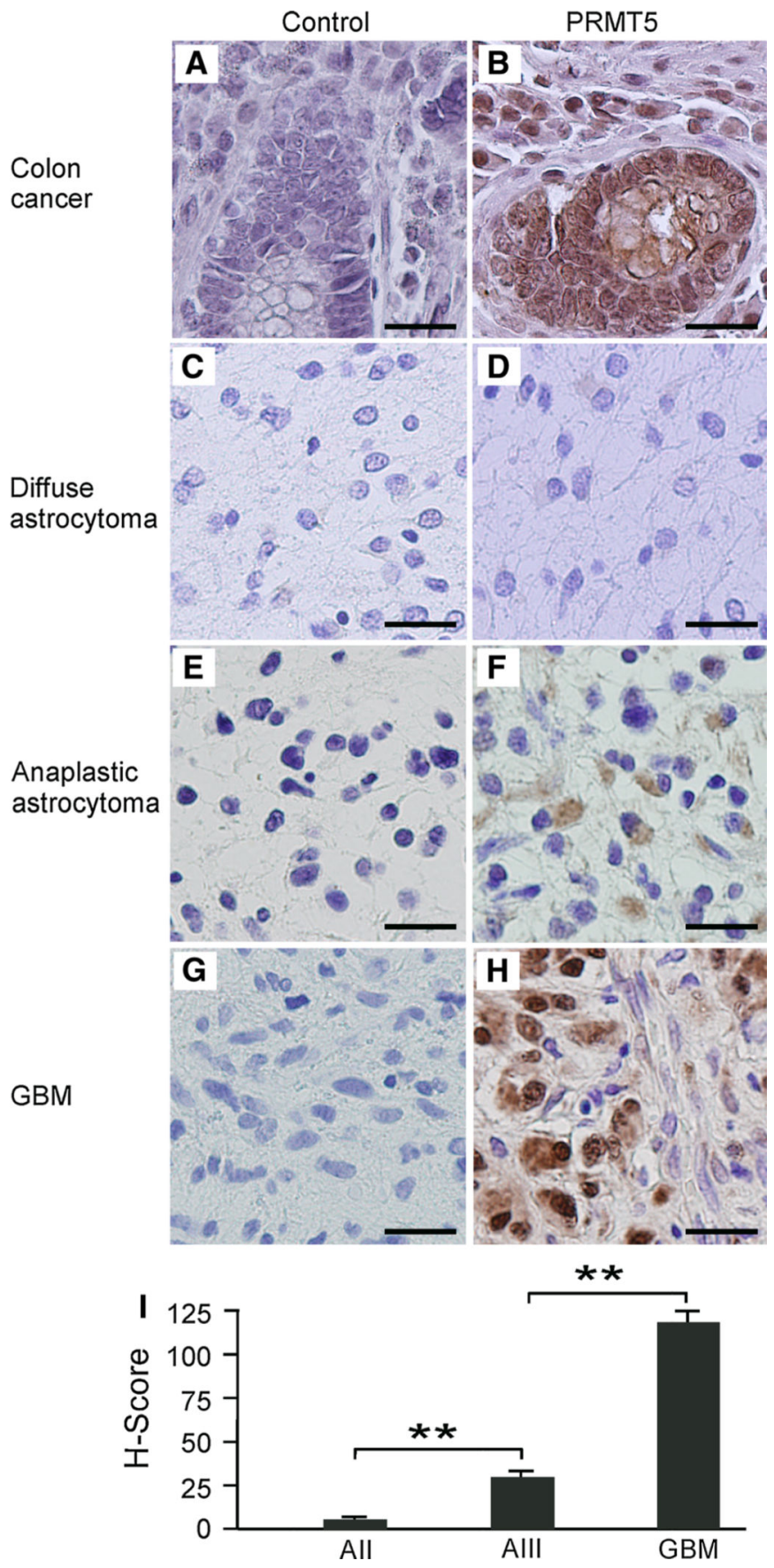

Fig. 4.

PRMT5 expression correlates with grade of malignancy in astroglial tumors. Scale bar 5 $\mu \mathrm{m}$. a, b PRMT5 stain in colon cancer (positive control). c, d PRMT5 was very low in WHO grade II astrocytomas. e, f Low levels of PRMT5 were detected in anaplastic astrocytomas. g, h PRMT5 immunoreactivity was present throughout glioblastoma cells and showed a nuclear predominance. i An H-Score was calculated based on intensity and the percentage of positively stained cells (see "Materials and Methods" section). The mean H-Score of GBM was significantly higher than that of anaplastic astrocytoma (AIII), which was significantly 
higher than that of diffuse astrocytoma (AII). ${ }^{* *} p<0.01$ by one-way ANOVA with post hoc Tukey test 

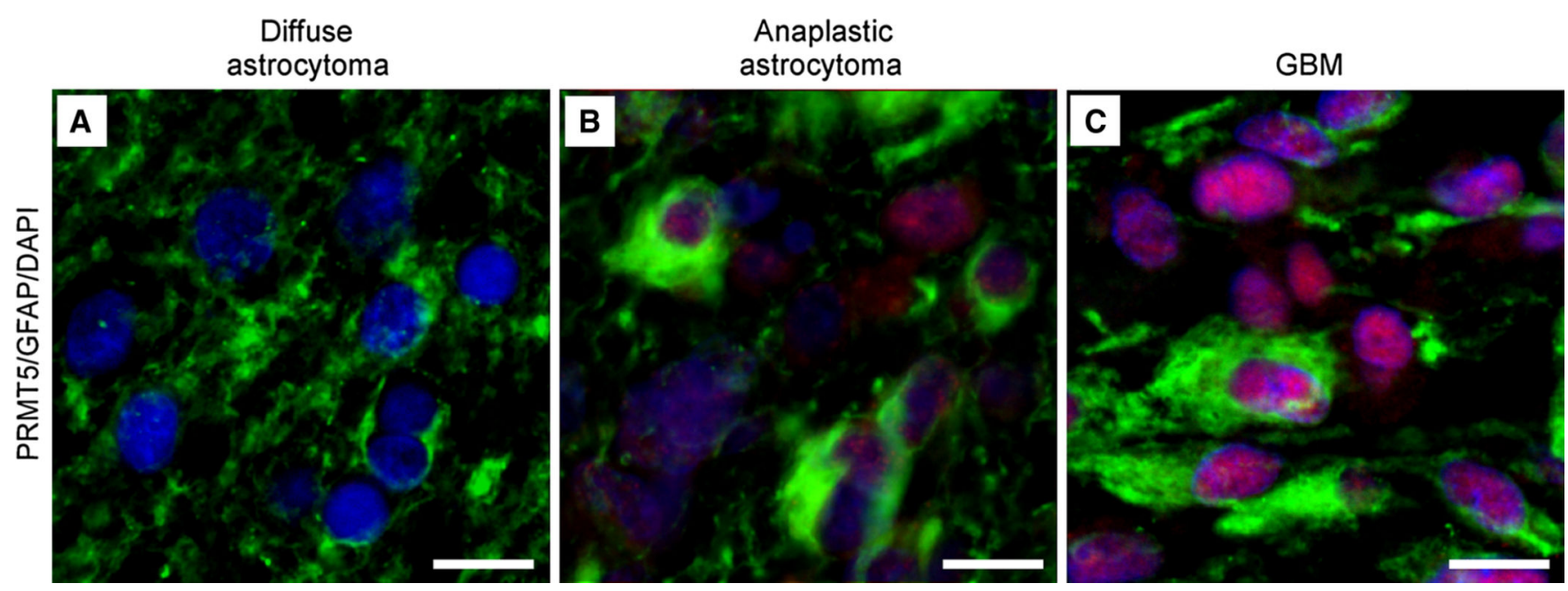

Fig. 5.

Immunofluorescent staining showed co-localization of PRMT5 and GFAP in different grades of astrocytomas. Sections of different grades of astrocytomas were immunostained with anti-PRMT5 (red), anti-GFAP (green) and propidium iodide. Scale bar $2 \mu \mathrm{m}$. a PRMT5 stain was very low in diffuse astrocytomas. b Moderate PRMT5 signals were detected in specimens of anaplastic astrocytomas. $\mathbf{c}$ GBM cells stained positive for PRMT5, with a nuclear concentration 
A

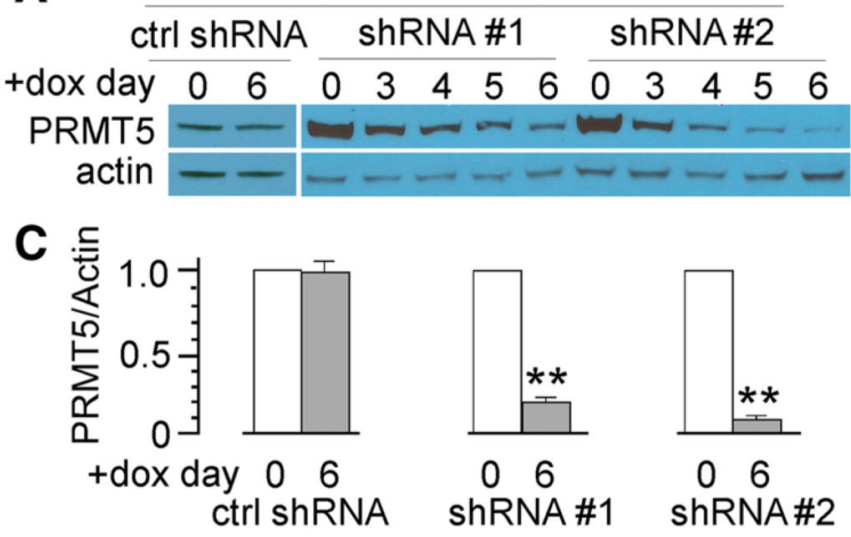

$\mathbf{E}$
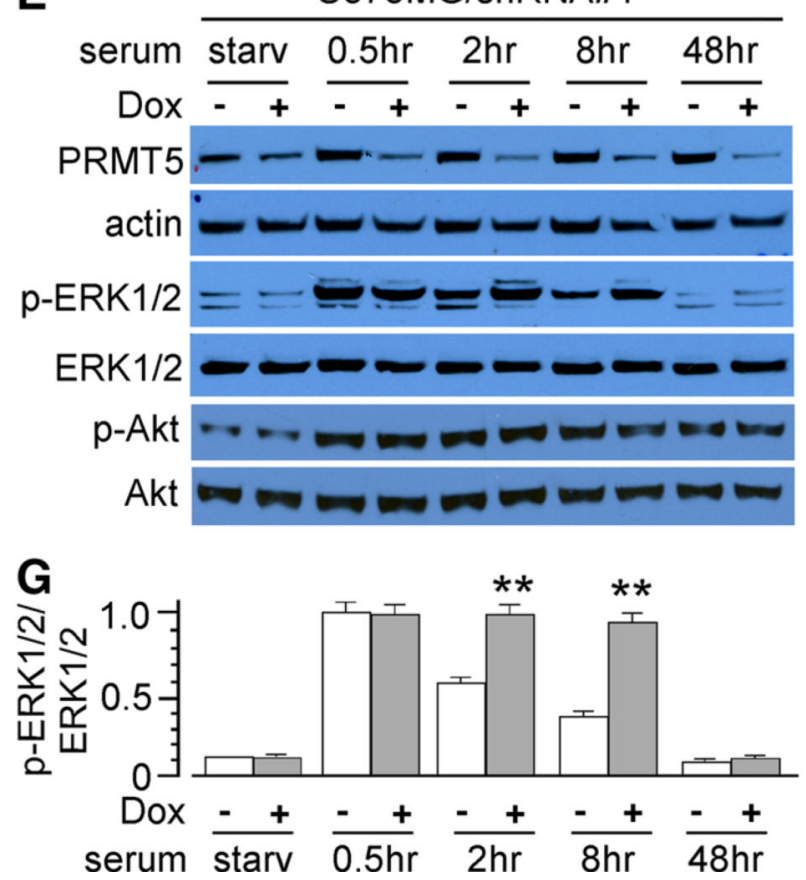

B

LN229
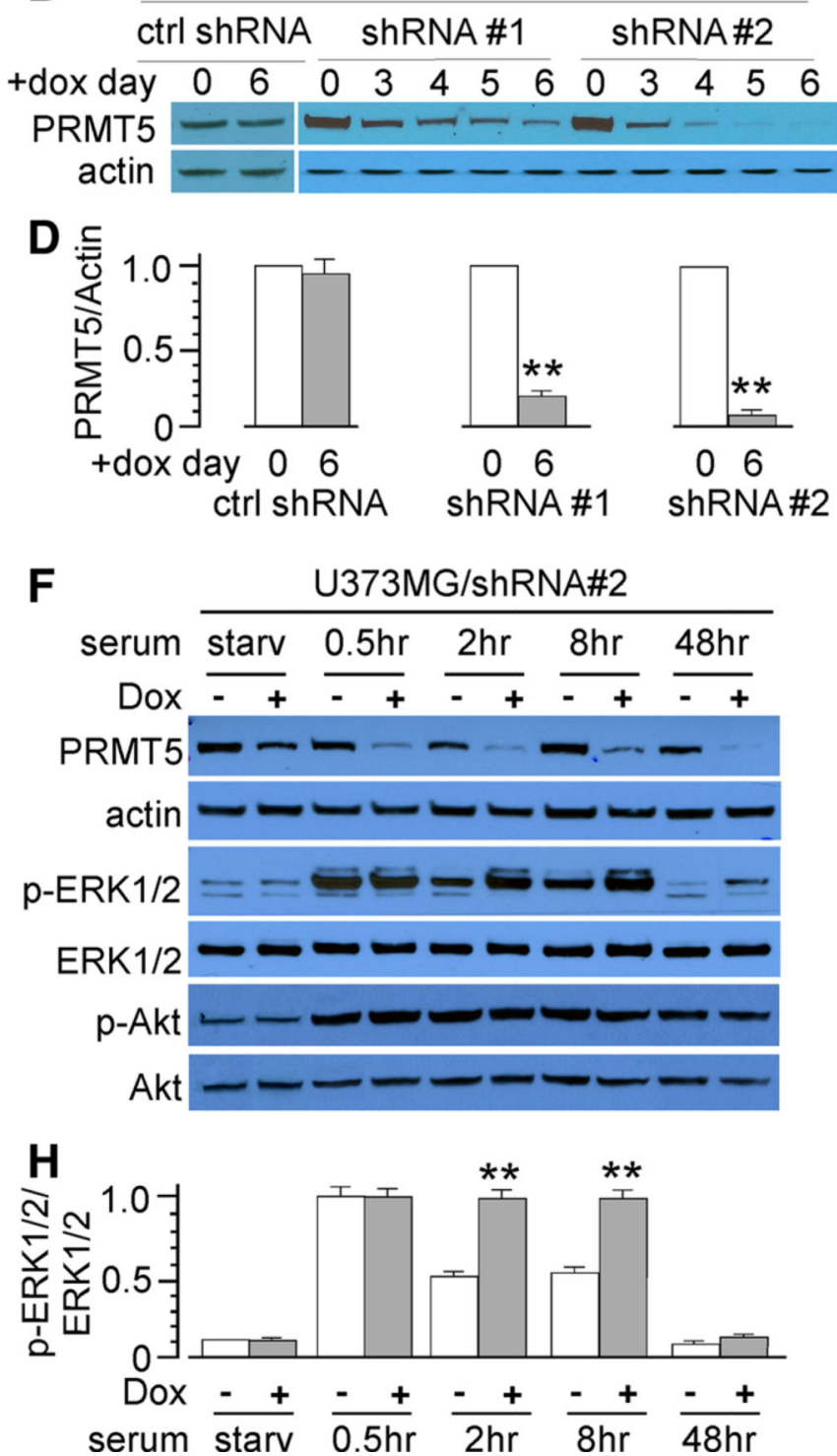

Fig. 6.

Knockdown of PRMT5 by tet-inducible shRNAs causes prolonged activation of ERK1/2. a, b Glioma cell lines U373MG and LN229 were transduced with lentiviruses containing doxycycline inducible scrambled shRNA or shRNA targeting two different sites in the PRMT5 mRNA (shRNA\#1 and 2). PRMT5 expression was determined by western blot after induction with doxycycline. $\mathbf{c}$ The amount of PRMT5 was unchanged by control shRNA. The levels of PRMT5 were significantly reduced to less than 20 and $10 \%$ (day 6) by shRNA\#1 and \#2 as measured by densitometry (normalized to actin). $\mathbf{d}$ Inducible knockdown of PRMT5 by shRNA\#1 and \#2 was achieved in LN229. e, $\mathbf{g}$ Knockdown of PRMT5 by shRNA\#1 changed the activation dynamics of p-ERK1/2. Cells grown with or without doxycycline were serum starved for $24 \mathrm{~h}$ and then exposed to serum (9\% FBS), and ERK activation was analyzed. In the controls, p-ERK1/2 peaked at $30 \mathrm{~min}$ and then declined 
to baseline at $48 \mathrm{~h}$ after exposure to serum. With PRMT5 knockdown, sustained peak levels of p-ERK1/2 were observed for up to $8 \mathrm{~h}$. The extent Akt phosphorylation was not affected by PRMT5 knockdown. $\mathbf{f}, \mathbf{h}$ Knockdown of PRMT5 by shRNA\#2 also caused prolonged high levels of $\mathrm{p}$-ERK1/2 without affecting p-Akt. $* * p<0.01$ 

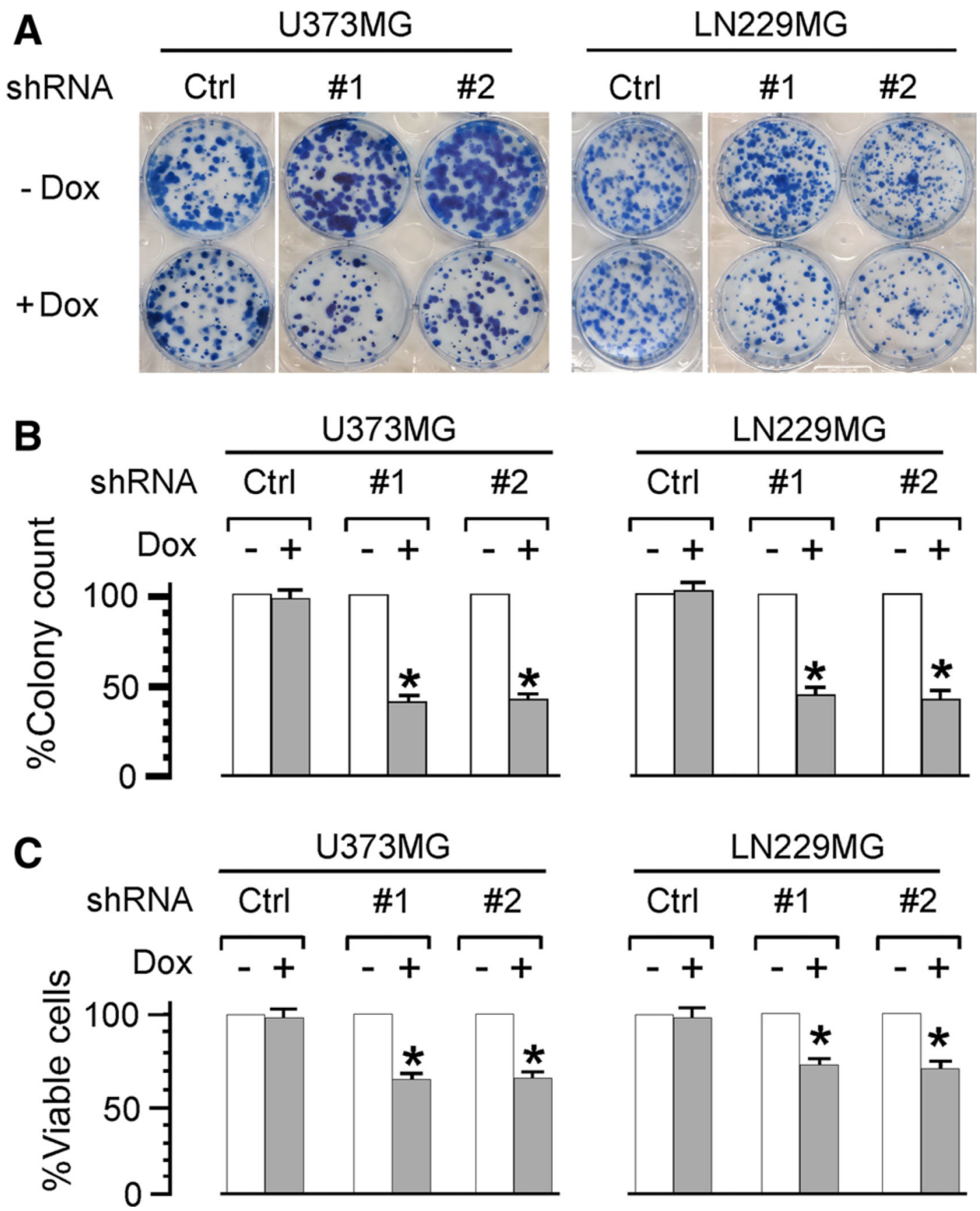

Fig. 7.

Knockdown of PRMT5 inhibits clonogenic formation and cell growth. a Lentivirus transduced cell lines were grown in the presence or absence of doxycycline for 2 weeks, and the colonies were fixed and stained. b Colonies of 20 cells or more were counted and averages were compared to the control. Doxycycline induction significantly reduced the number of colonies formed. cell viability after PRMT5 knockdown was assessed by the MTS cell viability assay after 4 days of doxycycline induction. Viable cells were 
significantly reduced in the presence of doxycycline. Experiments were performed in triplicate and repeated three times. ${ }^{*} p<0.01$ 
